\title{
PRINCIPAL ROLE OF DIHYDROPTEROATE SYNTHASE MUTATIONS IN MEDIATING RESISTANCE TO SULFADOXINE-PYRIMETHAMINE IN SINGLE-DRUG AND COMBINATION THERAPY OF UNCOMPLICATED MALARIA IN UGANDA
}

\author{
GRANT DORSEY, CHRISTIAN DOKOMAJILAR, MOSES KIGGUNDU, SARAH G. STAEDKE, MOSES R. KAMYA, AND \\ PHILIP J. ROSENTHAL \\ Department of Medicine, San Francisco General Hospital, University of California, San Francisco, California; Makerere University \\ Medical School, Kampala, Uganda
}

\begin{abstract}
Antimalarial resistance to sulfadoxine-pyrimethamine (SP) is mediated by mutations in the dihydrofolate reductase $(d h f r)$ and dihydropteroate synthase (dhps) genes. However, the relative importance of different mutations is incompletely understood and has not been studied with combination therapy. Samples from 812 patients treated for uncomplicated malaria in Kampala, Uganda were tested for the presence of mutations commonly found in Africa. The dhps Glu-540 mutation was the strongest independent predictor of treatment failure. The dhfr Arg-59 mutation was only predictive of treatment failure in the presence of the dhps Glu-540 mutation. Comparing combination regimens with SP monotherapy, the addition of chloroquine to SP did not improve efficacy, the addition of artesunate lowered the risk of treatment failure only for infections with both the dhfr Arg-59 and dhps Glu-540 mutations, and the addition of amodiaquine lowered this risk for all $d h f r / d h p s$ mutation patterns. The dhps Glu-540 mutation played a principal role and the $d h f r$ Arg-59 mutation a secondary role in mediating resistance to SP alone and in combination.
\end{abstract}

\section{INTRODUCTION}

Sulfadoxine-pyrimethamine (SP) is currently the first-line antimalarial therapy in several African countries where chloroquine $(\mathrm{CQ})$ resistance is widespread and other drugs remain too expensive for general use. ${ }^{1}$ It is generally regarded as a single antimalarial agent because its success depends on the synergistic action of its two component inhibitors of folate synthesis. ${ }^{2}$ Resistance to SP spread rapidly in southeast Asia following widespread use ${ }^{3}$ and is now spreading in Africa. ${ }^{4}$ In an attempt to improve treatment efficacy and delay the spread of drug resistance, several countries in Africa recently changed to inexpensive combinations of SP plus CQ or amodiaquine (AQ) as first-line antimalarial therapy. ${ }^{1}$ In addition, many authorities advocate combinations with artesunate (AS) or other artemisinins, including SP plus AS, ${ }^{5}$ although these regimens are expensive.

Resistance to SP is caused by point mutations that accumulate at several sites in the dihydrofolate reductase $(d h f r)$ and dihydropteroate synthase (dhps) genes, resulting in increasing degrees of drug resistance in vitro. ${ }^{2,6}$ For $d h f r$, a point mutation at position 108 (Ser $\rightarrow$ Asn) increases resistance to pyrimethamine approximately 100 -fold. ${ }^{7}$ The addition of mutations at positions 51 (Asn $\rightarrow$ Ile) and 59 (Cys $\rightarrow$ Arg) progressively enhances in vitro resistance to pyrimethamine. ${ }^{2}$ The relationship between dhps mutations and in vitro resistance to sulfadoxine is less clear, in part because the in vitro measurement of sulfadoxine activity is complex due to differences in assay conditions and variations in the ability of different parasite strains to use exogenous folate. ${ }^{2,6,8}$ Nevertheless, it appears that in vitro resistance to sulfadoxine is associated with accumulation of point mutations in the dhps gene. ${ }^{9}$

Assessment of molecular markers of SP resistance has been proposed as a means of monitoring in vivo drug resistance. However, many questions remain about the relationship between $d h f r$ and dhps mutations that confer in vitro resistance and clinical treatment outcomes. The $d h f r$ Asn-108 mutation alone does not appear to be sufficient to confer clinical drug resistance. ${ }^{10}$ The $d h f r$ triple mutant (Asn-108 + Ile-51 + Arg-
59) has been suggested as a useful predictor of treatment failure by some investigators, ${ }^{11,12}$ although others have not found useful predictive value for this genotype. ${ }^{13,14}$ Others have suggested that the most useful predictor of SP treatment failure is a combination of the $d h f r$ triple mutant and two dhps mutations (Gly-437 + Glu-540). ${ }^{15,16}$ A better understanding of the relationship of key $d h f r$ and $d h p s$ mutations in field isolates and of the independent roles of each mutation in mediating response to therapy will be helpful in directing the rational use of SP and SP-containing combination regimens.

In this study, we evaluated the relationship between key dhfr and dhps mutations in a large sample of patients $(\mathrm{n}=$ 812) with uncomplicated Plasmodium falciparum malaria from three clinical trials conducted over a five-year period in Kampala, Uganda. In addition, we evaluated combinations of $d h f r$ and dhps alleles to identify the strongest predictors of treatment failure, and we compared the relationship between allelic risk groups and response to SP monotherapy and three $\mathrm{SP}$ combination therapies $(\mathrm{SP}+\mathrm{CQ}, \mathrm{SP}+\mathrm{AQ}$, and $\mathrm{SP}+\mathrm{AS})$.

\section{MATERIALS AND METHODS}

Study site and subjects. All studies were conducted between March 1999 and June 2003 at the outpatient department of Mulago Hospital in Kampala, the urban capital of Uganda. Malaria is mesoendemic in Kampala, with peak transmission occurring during two rainy seasons (Ugandan Ministry of Health, unpublished data). Patients included in this analysis came from three previously published clinical trials. The treatment arms and study dates are as follows: study 1; CQ versus SP, March 1999 to August $1999^{17}$; study 2; SP versus SP + AQ versus SP + AS, July 2000 to August $2001^{18}$; and study $3 ; \mathrm{SP}+\mathrm{CQ}$ versus $\mathrm{SP}+\mathrm{AQ}$ versus $\mathrm{AQ}+$ AS, August 2002 to July 2003 (Staedke SG, unpublished data). Only patients enrolled in SP monotherapy arms or SP combination therapy arms $(\mathrm{SP}+\mathrm{CQ}, \mathrm{SP}+\mathrm{AQ}$, or $\mathrm{SP}+\mathrm{AS})$ fulfilling the following selection criteria were included in this study: 1) age range $=6$ months to 10 years, 2) tympanic temperature $\geq 38^{\circ} \mathrm{C}$ or a history of fever in the previous 48 
hours, 3) absence of severe malaria ${ }^{19}$ or danger signs (inability to stand or drink, lethargy, recent convulsions, persistent vomiting), 4) willingness to provide informed consent, 5) residence within Kampala 6) $P$. falciparum mono-infection, 7) parasite density $\geq 500$ asexual parasites/ $\mu \mathrm{L}$, and 8) successful outcome classification after 28 days of follow-up, adjusted for genotyping.

Informed consent was obtained from all adult participants and from parents or legal guardians of minors. All protocols were reviewed and approved by the Institutional Review Boards of Makerere University, Kampala and the University of California, San Francisco.

Patient follow-up and outcome classification. Standardized clinical outcomes for all subjects were assessed using the 1996 World Health Organization (WHO) clinical classification system (adequate clinical response [ACR], early treatment failure $[\mathrm{ETF}]$, or late treatment failure $[\mathrm{LTF}])^{20}$ with follow-up extended to 28 days and the modification that after day 3, patients with parasitemia and a history of recent fever (not documented) were considered LTF. For subjects with recurrent parasitemia after day 4 , pretreatment samples and samples from the day of treatment failure were analyzed for polymorphisms in merozoite surface protein 2 to distinguish recrudescence from new infections, according to previously published methods. ${ }^{21}$ Genotyping was successful in $97 \%$ of the samples analyzed. For the purposes of this study, treatment failure was defined as fulfillment of the WHO criteria for ETF or LTF in the presence of recrudescent parasites.

Mutation analysis. The presence of $d h f r$ Asn-108, Ile-51, and Arg-59 and dhps Gly-437 and Glu-540 mutations commonly found in Africa were determined using a nested polymerase chain reaction amplification followed by restriction enzyme digestion. All five mutations were assessed in the SP monotherapy group and only the dhfr Arg-59 and dhps Gly437 and Glu-540 mutations were assessed in the combination therapy groups. Blood was collected on filter paper on the day of diagnosis and parasite DNA isolated using the Chelex extraction method. ${ }^{22}$ Primers, amplification conditions, and restriction endonucleases for assays to detect all mutations were as previously described. ${ }^{15,23}$ Digestion products were visualized by gel electrophoresis and results were classified as wild-type, pure mutant, or mixed (both wild-type and mutant alleles present in the same infection) based on the migration patterns of the fragments. Investigators were blinded to clinical outcomes at the time of molecular analysis.
Statistical analysis. Associations between categorical variables were assessed using the chi-square or Fisher's exact test as appropriate. Associations between combinations of $d h f r$ and $d h p s$ mutations and treatment failure were assessed using multivariate logistic regression, controlling for age and parasite density, with treatment and allelic risk groups included as interaction terms. All interaction terms with $P$ values $\leq 0.2$ were retained in the final model. Graphic presentation of the change in the prevalence of mutations over calendar time was generated using the lowess locally weighted regression smoothing technique. Parasite density was normalized using natural $\log$ transformation. All data were entered and verified using Epi-Info version 6.04 (Centers for Disease Control and Prevention, Atlanta, GA) and SPSS (SPSS Inc., Chicago, IL). Analysis was performed using STATA statistical software (Stata Corp., College Station, TX). All confidence levels were set at $95 \%$. Data on the association between molecular markers of SP resistance and clinical outcomes in the SP monotherapy group have been published previously, ${ }^{15,24}$ but were included in this study to improve statistical power and provide a comparison group for the SP-combination therapies.

\section{RESULTS}

Patient characteristics and treatment outcomes. A total of 812 subjects from 4 different treatment groups enrolled between March 1999 and April 2003 were included in this study. Compared with the SP monotherapy group (32\%), the risk of treatment failure at 28 days was similar in the SP + CQ group (35\%; $P=0.58)$ and significantly lower in the SP + AS (17\%; $P=0.0003)$ and SP + AQ $(7 \% ; P<0.0001)$ groups (Table 1$)$.

Prevalence and relationship of $\boldsymbol{d h f r}$ and $\boldsymbol{d h p s}$ mutations. We were interested in the prevalence of key $d h f r$ (Asn-108, Ile-51, and Arg-59) and dhps mutations (Gly-437 and Glu-540) and the relationship between these mutations. A total of 2,832 (97\%) of 2,930 molecular assays were successfully performed for $d h f r$ Asn-108 and Ile-51 mutations in the SP monotherapy group and for dhfr Arg-59 and dhps Gly-437 and Glu-540 mutations in all treatment groups. The dhfr Asn-108 and Ile-51 mutations were very common, with $88 \%$ and $91 \%$ of samples having the pure mutant and $97 \%$ and $94 \%$ having a mixed or pure mutant, respectively (Figure $1)$. The $d h f r$ Arg-59 mutation occurred less commonly and was strongly linked to the $d h f r$ Asn-108 and Ile-51 mutations. Of the 161 samples that were mixed or pure mutant for $d h f r$ Arg-59, only 3 (2\%) were wild-type for $d h f r$ Asn-108, 6 (4\%)

TABLE 1

Baseline characteristics and treatment outcomes

\begin{tabular}{|c|c|c|c|c|}
\hline & \multicolumn{4}{|c|}{ Treatment group* } \\
\hline & SP & $\mathrm{SP}+\mathrm{CQ}$ & $\mathrm{SP}+\mathrm{AS}$ & $\mathrm{SP}+\mathrm{AQ}$ \\
\hline Number of subjects & 247 & 125 & 173 & 267 \\
\hline \multirow[t]{2}{*}{ Studies (n) and enrollment period $\dagger$} & Study 1 (63) & Study 3 (125) & Study 2 (173) & Study 2 (138) \\
\hline & Study 2 (184) & & & Study 3 (129) \\
\hline Median age in years (range) & $3.1(0.5-10)$ & $4.3(0.5-10)$ & $3.1(0.6-5.7)$ & $3.1(0.5-10)$ \\
\hline Geometric mean parasite density (per $\mu \mathrm{l})$ & 35,027 & 24,191 & 52,727 & 36,446 \\
\hline Early treatment failure $\neq(\%)$ & $25(10 \%)$ & $2(2 \%)$ & 0 & $5(2 \%)$ \\
\hline Late treatment failures $\S(\%)$ & $55(22 \%)$ & $42(34 \%)$ & $29(17 \%)$ & $14(5 \%)$ \\
\hline
\end{tabular}

$* \mathrm{SP}=$ sulfadoxine/pyrimethamine; $\mathrm{CQ}=$ chloroquine; $\mathrm{AS}=$ artesunate; $\mathrm{AQ}=$ amodiaquine.

$\dagger$ Study 1 March 1999-August 1999; Study 2 July 2000-August 2001; Study 3 August 2002-April 2003.

$\doteqdot$ Treatment failure on days $1-3$.

$\S$ Treatment failure on days $4-28$ adjusted by genotyping. 


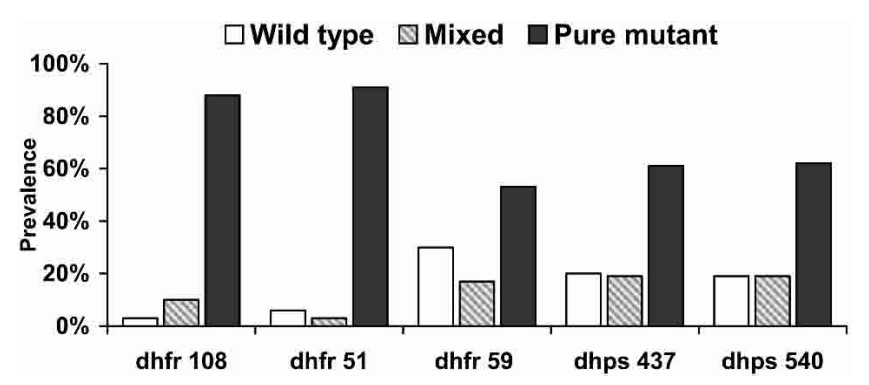

FiguRE 1. Prevalence of key dihydrofolate reductase (dhfr) and dihydropteroate synthetase (dhps) mutations for all treatment groups combined.

were wild-type for $d h f r$ Ile-51, and none were wild-type for both. The prevalences of the dhps Gly-437 and Glu-540 mutations were $61 \%$ and $62 \%$ for the pure mutant and $80 \%$ and $81 \%$ for the mixed or pure mutant, respectively (Figure 1). The dhps Gly-437 and Glu-540 alleles were strongly linked, with $641(82 \%)$ of 778 samples having the same result and only 56 (7\%) of 778 samples wild-type for one dhps allele and mixed or pure mutant for the other. Considering relationships between mutations in different genes, there was a significant association between the dhfr Arg-59 and dhps Glu-540 mutations $(P<0.001)$. However, $94(12 \%)$ of 782 samples contained the dhfr Arg-59 mutation in the absence of the dhps Glu-540 mutation and 181 (23\%) of 782 samples contained the dhps Glu-540 in the absence of the dhfr Arg-59 mutation. Thus, it was not uncommon for the dhps Glu-540 mutation to occur in the absence of the dhfr Arg-59 mutation.

Association between $\boldsymbol{d h} f \boldsymbol{r}$ and $\boldsymbol{d h p s}$ alleles and treatment outcomes. To maximize our sample size, we pooled the treatment groups from our three clinical studies and evaluated the association between $d h f r$ and dhps alleles and response to therapy. To simplify our analysis, we focused only on those alleles that were independent predictors of treatment outcome. Given the very high prevalence of the $d h f r$ Asn-108 and Ile-51 mutations and their almost ubiquitous presence in the setting of the $d h f r$ Arg-59 mutation, results at the dhfr 108 and 51 alleles did not add to the predictive value of the dhfr 59 allele alone and were thus not assessed for combination therapy groups. Given the strong association between the dhps 437 and 540 alleles, we evaluated their individual predictive values. Considering samples that were dhps Gly-437 mixed or pure mutant and dhps 540 wild-type, only 1 $(4 \%)$ of 23 resulted in treatment failure. In contrast, considering samples that were dhps Glu-540 mixed or pure mutant and dhps 437 wild-type, 8 (24\%) of 33 resulted in treatment failure. Based on these findings, we concluded that results for the dhps 437 allele did not add to the predictive value of the dhps 540 allele alone.

Since $d h f r 108$ and 51 alleles and the dhps 437 allele were not independent predictors, further analyses concentrated on the association between dhfr 59 and dhps 540 alleles and treatment outcomes. Our goal was to identify patterns that best predicted treatment outcomes based on all possible combinations of these two alleles. In this and all subsequent analyses, ETFs were excluded because we previously showed that molecular markers of SP resistance were not associated with $\mathrm{ETFs}^{15}$; others have suggested that many ETFs are due to slow clearance of parasites without true parasite resistance. ${ }^{16}$
Risk of treatment failure was assessed based on $d h f r 59$ and dhps 540 alleles. For samples containing the dhps 540 wildtype allele (termed the low-risk allelic group), the risk of treatment failure was low ( 6 of $143,4 \%$ ), and this risk was not significantly different across strata based on $d h f r 59$ alleles $(P=0.38)$ (Table 2). For samples containing the dhps Glu540 mixed or pure mutant allele, but excluding $d h f r$ Arg-59 + dhps Glu-540 pure mutants (termed the intermediate-risk allelic group), the risk of treatment failure was significantly higher (58 of 327, 18\%) compared with the low-risk group ( $P$ $=0.0001)$. Again, there was no significant difference in the risk of treatment failure for this group across strata based on dhfr 59 alleles $(P=0.80)$ (Table 2). For samples containing both the dhfr Arg-59 and dhps Glu-540 pure mutants (termed the high-risk allelic group), the risk of treatment failure was significantly higher (76 of $283,27 \%$ ) compared with the low risk $(P<0.0001)$ and the intermediate risk allelic groups $(P=$ $0.007)$. Thus, the presence of the $d h f r$ Arg-59 mutation had an independent effect on the risk of treatment failure only in the presence of the dhps Glu-540 pure mutant. In contrast, the presence of the dhps Glu-540 mutation had an independent effect on the risk of treatment failure even in the absence of the dhfr Arg-59 mutation.

Interestingly, the prevalence of the dhfr Arg-59 and dhps Glu-540 pure mutants increased over the four-year period during which samples were collected (Figure 2). The average prevalence of the dhfr Arg-59 pure mutant increased from $40 \%$ in 1999 to $60 \%$ in 2003 and the average prevalence of the dhps Glu-540 pure mutant increased from 40\% in 1999 to $70 \%$ in 2003 .

Association between $\boldsymbol{d h f r}$ and $d h p s$ alleles and treatment outcomes stratified by treatment group. For SP monotherapy, the risk of treatment failure was $7 \%$ for the low-risk allelic group, $23 \%$ for the intermediate-risk group, and $37 \%$ for the high-risk group (Figure 3 ). When age and pretreatment parasite density were controlled, differences in these associations were statistically significant (intermediate- versus low-risk allelic groups, odds ratio $[\mathrm{OR}]=6.3, P<0.001$; high- versus low-risk allelic groups, $\mathrm{OR}=14.4, P<0.001)($ Table 3$)$. Compared with SP monotherapy, SP + CQ combination therapy was associated with a similar relationship between the dhfr 59 and dhps 540 allelic risk groups and the risk of treatment failure (Figure 3 and Table 3). Combining AS with

TABLE 2

Association between $d h f r 59$ and dhps 540 alleles and late treatment failure*

\begin{tabular}{|c|c|c|c|c|}
\hline $\begin{array}{l}d h f r 59 \\
\text { Allele }\end{array}$ & $\begin{array}{l}\text { dhps } 540 \\
\text { Allele }\end{array}$ & $\mathrm{N}$ & $\begin{array}{c}\text { Risk of late } \\
\text { treatment failure } \dagger\end{array}$ & Assigned risk group \\
\hline Wild & Wild & 52 & $2 \%$ & Low-risk group $\ddagger$ \\
\hline Mixed & Wild & 22 & $9 \%$ & \\
\hline Mutant & Wild & 69 & $4 \%$ & \\
\hline Wild & Mixed & 41 & $20 \%$ & Intermediate-risk group $\S$ \\
\hline Mixed & Mixed & 61 & $21 \%$ & \\
\hline Mutant & Mixed & 43 & $19 \%$ & \\
\hline Wild & Mutant & 135 & $16 \%$ & \\
\hline Mixed & Mutant & 47 & $17 \%$ & \\
\hline Mutant & Mutant & 283 & $27 \%$ & High-risk groupd \\
\hline
\end{tabular}




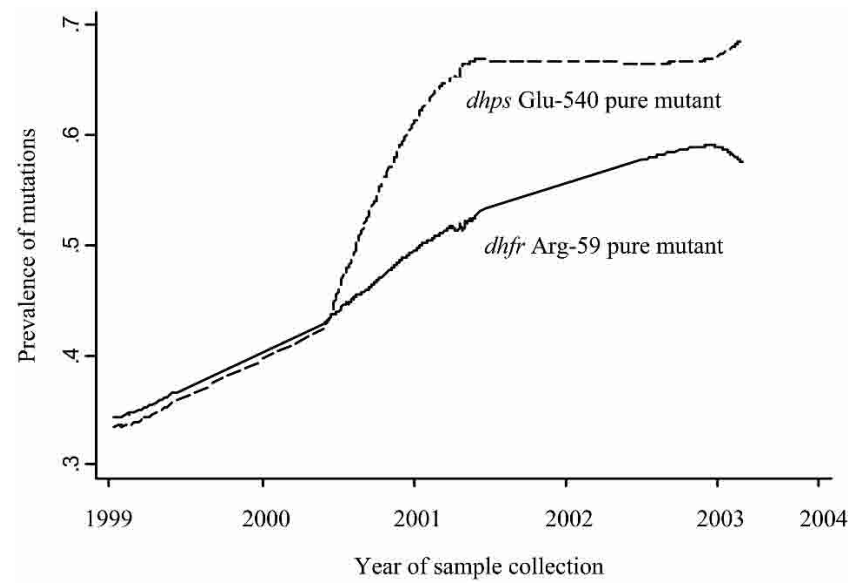

FIGURE 2. Change in average prevalence of dihydrofolate reductase (dhfr) Arg-59 and dihydropteroate synthetase (dhps) Glu-540 pure mutants over the duration of the three clinical trials using the lowess locally weighted regression smoothing technique. Study 1 (sulfadoxine-pyrimethamine [SP] monotherapy), March 1999 to August 1999; study 2 (SP monotherapy, SP plus amodiaquine [AQ] and SP + artesunate [AS]), July 2000 to August 2001; study 3 (SP plus CQ and SP plus AQ), August 2002 to July 2003.

SP resulted in an overall reduction in the risk of treatment failure (Figure 3), and the association between dhfr Arg-59 and dhps Glu-540 mutations and treatment failure remained statistically significant (Table 3). However, samples from the intermediate and high-risk groups had similar risks of treatment failure $(\mathrm{OR}=6.3, P<0.001$ and $\mathrm{OR}=5.3, P=0.002$, respectively). Combining $\mathrm{AQ}$ with $\mathrm{SP}$ also resulted in an overall reduction in the risk of treatment failure (Figure 3); however, compared with samples from the low-risk allelic group, only samples from the high-risk allelic group were associated with treatment failure $(\mathrm{OR}=2.8, P=0.06)$ (Table 3 ). The associations between $d h f r$ and $d h p s$ allelic risk groups and treatment outcomes presented in Table 3 were not significantly different across different age groups (i.e., there was no evidence of effect modification by age).

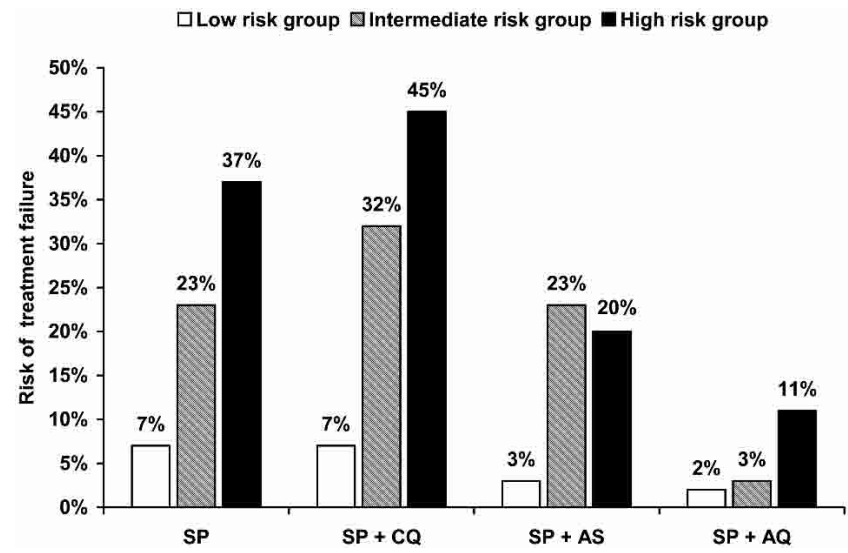

FIGURE 3. Treatment failure defined as a late treatment failure on days $4-28$ adjusted by genotyping. $\mathrm{SP}=$ sulfadoxine-pyrimethamine, $\mathrm{CQ}=$ chloroquine, $\mathrm{AQ}=$ amodiaquine, $\mathrm{AS}=$ artesunate. Low-risk group $=$ dihydrofolate reductase $(d h f r) 59$ any genotype and dihydropteroate synthetase (dhps) 540 wild-type; intermediate-risk group = dhps Glu-540 mixed or pure mutant (excluding high-risk group); high-risk group $=d h f r$ Arg-59 pure mutant and dhps Glu-540 pure mutant.
TABLE 3

Association between $d h f r 59$ and dhps 540 alleles and late treatment failure for each treatment group*

\begin{tabular}{lccc}
\hline \multicolumn{1}{c}{ dhfr/dhps Allelic group } & Treatment group & OR $(95 \% \mathrm{CI}) \dagger$ & $P$ \\
\hline Low-risk group $\ddagger$ & All & 1.0 (reference) & - \\
\hline Intermediate-risk group\$ & SP & $6.3(2.6-15.5)$ & $<0.001$ \\
& SP + CQ & $13.0(4.7-35.7)$ & $<0.001$ \\
& SP + AS & $6.3(2.6-15.5)$ & $<0.001$ \\
& SP + AQ & $0.63(0.2-2.6)$ & 0.53 \\
\hline High risk-groupII & SP & $14.4(5.6-36.7)$ & $<0.001$ \\
& SP + CQ & $23.8(8.7-65.5)$ & $<0.001$ \\
& SP + AS & $5.3(1.8-15.3)$ & 0.002 \\
& SP + AQ & $2.8(1.0-8.0)$ & 0.06 \\
\hline
\end{tabular}

$* d h f r=$ dihydrofolate reductase; $d h p s=$ dihydropteroate synthase; SP $=$ sulfadoxine pyrimethamine; $\mathrm{CQ}=$ chloroquine; $\mathrm{AS}=$ artesunate; $\mathrm{AQ}=$ amodiaquine.

$\dagger$ Multivariate logistic regression controlling for age and pretreatment parasite density including significant interaction terms based on treatment and allelic risk groups $\mathrm{OR}=$ odd ratio; $\mathrm{CI}=$ confidence interval.

\$Low-risk group $=d h f r 59$ any genotype and dhps 540 wild-type

$\S$ Intermediate-risk group $=$ dhps Glu-540 mixed or pure mutant (excluding high-risk group).

qI High-risk group $=d h f r$ Arg-59 pure mutant and dhps Glu-540 pure mutant.

\section{DISCUSSION}

In this study, we investigated the relationship between key $d h f r$ and dhps mutations found in Africa and clinical response to SP therapy alone and in combination with other drugs. We developed a simplified predictive model of treatment response based only on the presence of the $d h f r$ Arg-59 and dhps Glu-540 mutations, largely due to the fact that the presence of these mutations was highly predictive of the $d h f r$ triple mutant (Asn-108 + Ile-51 + Arg-59) and the dhps double mutant (Gly-437 + Glu-540), respectively. Considering all treatment groups combined, the presence of the dhps Glu-540 mutation was a much stronger predictor of clinical treatment failure than the $d h f r$ Arg-59 mutation. Indeed, the dhfr Arg-59 mutation was only an independent predictor of treatment failure in the presence of the dhps Glu-540 pure mutant. Compared with SP monotherapy, the addition of CQ to SP did not improve the risk of treatment failure after controlling for the prevalence of the $d h f r / d h p s$ allelic risk groups. The addition of AS to SP only lowered the risk of treatment failure due to parasites containing both the $d h f r$ Arg-59 and dhps Glu-540 pure mutants. The addition of AQ to SP lowered the risk of treatment failure for all $d h f r / d h p s$ allelic risk groups, and for this combination therapy treatment failure was only associated with the $d h f r$ Arg-59 + dhps Glu-540 pure mutant.

Several studies from Africa have examined the relationship between key $d h f r$ and dhps mutations in field isolates. With respect to $d h f r$ mutations, it has been proposed that the selection of mutations occurs in a stepwise fashion, first with the selection of the Asn-108 mutation, followed by the selection of mutations Ile-51 and/or Arg-59. ${ }^{25}$ Data on the selection of dhps mutations is less clear. Some studies have reported the presence of the dhps Gly-437 mutation in the absence of the Glu-540 mutation $^{12,26}$ or the presence of the Glu-540 mutation only in combination with the Gly-437 mutation, ${ }^{27}$ suggesting that the Gly-437 mutation is selected first. ${ }^{25}$ However, the presence of the dhps Glu-540 mutation in the absence of the Gly-437 mutation has been reported, although it appears to be uncommon, ${ }^{16}$ as was the case in our study. It has been further suggested that common $d h f r$ and dhps mutations have arisen independently on numerous occasions, with the incremental selection of increasingly resistant parasites over 
time. ${ }^{28}$ However, recent data from South Africa and Tanzania demonstrated that gene flow, rather than the spontaneous generation of new mutations, has been the most common originator of SP resistance. ${ }^{4}$ In that study, a period of rapid deterioration of SP efficacy was linked to a high background prevalence of a single $d h f r$ triple mutant allele (Asn-108 + Ile-51 + Arg-59), followed by the spread of a single dhps double mutant allele (Gly-437 + Glu-540), each derived from independent ancestral lineages. The findings from our study of strong linkage between the three $d h f r$ mutations and, independently, between the two dhps mutations, which were substantially increasing in prevalence over the four-year span of our studies in Uganda, are consistent with this more recent understanding of the basis of spread of SP resistance.

The relative importance of $d h f r$ versus dhps mutations in mediating treatment failure to SP has not been clearly resolved. In Cameroon, the $d h f r$ triple mutant was associated with $7(100 \%)$ of 7 treatment failures, but was also present in $26(44 \%)$ of 59 treatment successes. ${ }^{12}$ In Gabon, the $d h f r$ triple mutant was associated with $9(82 \%)$ of 11 treatment failures, but was also present in $51(72 \%)$ of 71 treatment successes. ${ }^{14}$ In both of these studies, there was no clear association between dhps mutations and treatment failure, but importantly the dhps Glu-540 mutation was not detected at either site and despite the high prevalence of the $d h f r$ triple mutant $(50-73 \%)$, the 28 -day risk of SP treatment failure was less than $15 \%$. In studies from east Africa, where the reported risk of SP treatment failure is higher than in west Africa, infection with parasites containing both the $d h f r$ triple mutant (Asn-108 + Ile-51 + Arg-59) and the dhps double mutant (Gly-437 + Glu-540) was associated with the highest risk of treatment failure. ${ }^{16,27}$ However, due to relatively small samples sizes $(\mathrm{n}<100)$ and the highly concordant nature of $d h f r$ and dhps mutations in field isolates, previous studies have had limited power to evaluate independent associations between these mutations and treatment response. In our study, which benefited from a much larger sample size, we found that the dhps Glu-540 mutation (a surrogate marker of the Gly-437 + Glu-540 double mutant) was a strong predictor of treatment failure independent of the $d h f r$ Arg-59 mutation (a surrogate marker of the Asn-108 + Ile-51 + Arg-59 triple mutant). In addition, it was not uncommon $(23 \%$ of the samples) for the dhps Glu-540 mutation to occur in the absence of the $d h f r$ Arg-59 mutation, contradicting previous suggestions that key dhps mutations are rare in the absence of the $d h f r$ triple mutant. ${ }^{2}$ In contrast, the $d h f r$ Arg-59 mutation was associated with treatment failure only in the presence of the dhps Glu-540 mutation. These results are consistent with recent findings from South Africa, where five years after the establishment of the $d h f r$ triple mutant, an increase in the risk of SP treatment failure from $20 \%$ to $70 \%$ coincided with the emergence of the dhps double mutant. ${ }^{4}$

A recent detailed report summarized all known Ugandan drug efficacy studies from 1988 to 2000, many of which were previously unpublished, including studies with varied methodologies. ${ }^{29}$ Between 1988 and 1995, reported rates of parasitologic (RI-RIII) resistance in children up to 15 years of age were $2-58 \%$ for CQ and 0-4\% for SP. Between 1996 and 2000, rates of parasitologic resistance in children less than five years of age were $36-96 \%$ for CQ and $6-33 \%$ for SP. In addition, these more recent studies reported rates of clinical treatment failure of $10-81 \%$ for CQ and $3-25 \%$ for SP. In
2001, the Ugandan Ministry of Health replaced CQ with CQ + SP as first-line therapy for uncomplicated malaria.

Clinical response to antimalarial therapy involves a complex interaction between the parasite, drug, and host immune response. In this study, by restricting our analysis to children recruited from the same target population and controlling for age and parasite density, we were able to minimize the contribution of differences in the host immune response. This allowed us to directly compare associations between molecular markers of SP resistance and clinical response to therapy. The use of SP combination therapy reduced the risk of ETF compared with SP monotherapy $(10 \%$ versus $1 \% ; P<0.001)$, probably due to more rapid initial parasite clearance. Surprisingly, the addition of CQ to SP did not lower the risk of LTF compared with SP alone even after controlling for the prevalence of key $d h f r$ and $d h p s$ mutations. Overall, the addition of CQ to SP had no impact on efficacy, and CQ is clearly a poor companion drug for SP where resistance to both drugs is high. ${ }^{18,30}$ Of note, in a previous study from Kampala, the prevalence of the pfcrt Thr-76 mutation (which has been linked to CQ resistance) was $100 \%$, suggesting the ubiquitous nature of resistance to this drug in our patient population. ${ }^{31}$ The addition of AS to SP reduced the risk of LTF, but only in infections with the most resistant parasites (dhfr Arg-59+ dhps Glu-540 pure mutants). The addition of AQ to SP lowered the risk of LTF for all $d h f r / d h p s$ allelic risk groups, and LTF was only associated with the $d h f r$ Arg-59 + dhps Glu-540 pure mutant. In our patient population, the combination of $\mathrm{SP}+\mathrm{AQ}$ was the most effective regimen, but also resulted in the strongest selective pressure for parasites most resistant to SP because almost all treatment failures carried the $d h f r$ Arg$59+$ dhps Glu-540 pure mutant. Thus, concern remains about the useful therapeutic lifespan of $\mathrm{SP}+\mathrm{AQ}$ in areas where pre-existing resistance to SP is high.

In summary, field studies of molecular markers of SP resistance can be useful for improving our understanding of the determinants of the clinical response to therapy. A combination of the $d h f r$ Arg-59 mutation (a surrogate marker of the $d h f r$ triple mutant) and the dhps Glu-540 mutation (a surrogate marker of the dhps double mutant) is the strongest predictor of treatment failure in Africa. Contrary to some prior suggestions, in our study the dhps Glu-540 mutation played a greater role in response to therapy than that of the $d h f r$ Arg59 mutation. Since more expensive antimalarial regimens, such as artemisinin-based combination therapy, remain beyond the reach of most African countries, SP combination therapies have been adopted as first-line treatment in a number of African countries. A better understanding of the relationship between molecular markers of SP resistance and response to therapy should help in establishing rational combination therapy policies and in estimating the useful therapeutic life span of these regimens.

Received May 5, 2004. Accepted for publication July 15, 2004.

Acknowledgments: We thank the clinical study team (B. M. Karakire, Marx Dongo, Sam Nsobya, Christopher Bongole, Regina Nakafero, Denise Njama, Anne Gasasira, Arthur Mpimbaza, Bridget K. Nzarubara, Pauline Byakika, and Sarah Kibirango); the community leaders from the Kawempe Division of Kampala; and the study participants and their parents/guardians for their support.

Financial support: This study was supported by the Fogarty International Center/National Institutes of Health (TW00007, TW01506, and AI43301) and the United Nations Development Program/World 
Bank/World Health Organization Special Program for Research and Training in Tropical Diseases (TDR).

Authors' addresses: Grant Dorsey, Christian Dokomajilar, Sarah G. Staedke, and Philip J. Rosenthal. University of California at San Francisco, Parnassus Avenue, Box 0811 San Francisco, CA, 94143 Telephone: 415-206-4680 Fax: 415-648-8425, E-mail: grantd@itsa. ucsf.edu. Moses Kiggundu and Moses R. Kamya, Makerere University Medical School, Anatomy Building, Room C-443, Kampala, Uganda, Telephone: 256-41-251-387 Fax: 256-41-540-524.

\section{REFERENCES}

1. AFRO, Global AMDP Database. http://rbm.who.int/amdp/ amdp_afro.htm.

2. Sibley CH, Hyde JE, Sims PF, Plowe CV, Kublin JG, Mberu EK, Cowman AF, Winstanley PA, Watkins WM, Nzila AM, 2001. Pyrimethamine-sulfadoxine resistance in Plasmodium falciparum: what next? Trends Parasitol 17: 582-588.

3. White NJ, Nosten F, Looareesuwan S, Watkins WM, Marsh K, Snow RW, Kokwaro G, Ouma J, Hien TT, Molyneux ME, Taylor TE, Newbold CI, Ruebush TK, Danis M, Greenwood BM, Anderson RM, Olliaro P, 1999. Averting a malaria disaster. Lancet 353: 1965-1967.

4. Roper C, Pearce R, Bredenkamp B, Gumede J, Drakeley C, Mosha F, Chandramohan D, Sharp B, 2003. Antifolate antimalarial resistance in southeast Africa: a population-based analysis. Lancet 361: 1174-1181.

5. World Health Organization, Position of WHO's Roll Back Malaria Department on Malaria Treatment Policy. http:// www.emro.who.int/rbm/.

6. Wang P, Read M, Sims PF, Hyde JE, 1997. Sulfadoxine resistance in the human malaria parasite Plasmodium falciparum is determined by mutations in dihydropteroate synthetase and an additional factor associated with folate utilization. Mol Microbiol 23: 979-986.

7. Peterson DS, Walliker D, Wellems TE, 1988. Evidence that a point mutation in dihydrofolate reductase-thymidylate synthase confers resistance to pyrimethamine in falciparum malaria. Proc Natl Acad Sci USA 85: 9114-9118.

8. Wang P, Sims PF, Hyde JE, 1997. A modified in vitro sulfadoxine susceptibility assay for Plasmodium falciparum suitable for investigating Fansidar resistance. Parasitology 115: 223-230.

9. Triglia T, Menting JG, Wilson C, Cowman AF, 1997. Mutations in dihydropteroate synthase are responsible for sulfone and sulfonamide resistance in Plasmodium falciparum. Proc Natl Acad Sci USA 94: 13944-13949.

10. Rallon NI, Osorio LE, Giraldo LE, 1999. Lack of an association between the ASN-108 mutation in the dihydrofolate reductase gene and in vivo resistance to sulfadoxine/pyrimethamine in Plasmodium falciparum. Am J Trop Med Hyg 61: 245-248.

11. Nzila AM, Nduati E, Mberu EK, Hopkins Sibley C, Monks SA, Winstanley PA, Watkins WM, 2000. Molecular evidence of greater selective pressure for drug resistance exerted by the long-acting antifolate pyrimethamine/sulfadoxine compared with the shorter-acting chlorproguanil/dapsone on Kenyan Plasmodium falciparum. J Infect Dis 181: 2023-2028.

12. Basco LK, Tahar R, Keundjian A, Ringwald P, 2000. Sequence variations in the genes encoding dihydropteroate synthase and dihydrofolate reductase and clinical response to sulfadoxinepyrimethamine in patients with acute uncomplicated falciparum malaria. J Infect Dis 182: 624-628.

13. Alifrangis M, Enosse S, Khalil IF, Tarimo DS, Lemnge MM, Thompson R, Bygbjerg IC, Ronn AM, 2003. Prediction of Plasmodium falciparum resistance to sulfadoxine/pyrimethamine in vivo by mutations in the dihydrofolate reductase and dihydropteroate synthetase genes: a comparative study between sites of differing endemicity. Am J Trop Med Hyg 69: 601-606.

14. Aubouy A, Jafari S, Huart V, Migot-Nabias F, Mayombo J, Durand R, Bakary M, Le Bras J, Deloron P, 2003. DHFR and DHPS genotypes of Plasmodium falciparum isolates from Gabon correlate with in vitro activity of pyrimethamine and cycloguanil, but not with sulfadoxine-pyrimethamine treatment efficacy. J Antimicrob Chemother 52: 43-49.
15. Kyabayinze D, Cattamanchi A, Kamya MR, Rosenthal PJ, Dorsey G, 2003. Validation of a simplified method for using molecular markers to predict sulfadoxine-pyrimethamine treatment failure in African children with falciparum malaria. Am J Trop Med Hyg 69: 247-252.

16. Kublin JG, Dzinjalamala FK, Kamwendo DD, Malkin EM, Cortese JF, Martino LM, Mukadam RA, Rogerson SJ, Lescano AG, Molyneux ME, Winstanley PA, Chimpeni P, Taylor TE, Plowe CV, 2002. Molecular markers for failure of sulfadoxinepyrimethamine and chlorproguanil-dapsone treatment of Plasmodium falciparum malaria. I Infect Dis 185: 380-388.

17. Kamya MR, Dorsey G, Gasasira A, Ndeezi G, Babirye JN Staedke SG, Rosenthal PJ, 2001. The comparative efficacy of chloroquine and sulfadoxine-pyrimethamine for the treatment of uncomplicated falciparum malaria in Kampala, Uganda. Trans $R$ Soc Trop Med Hyg 95: 50-55.

18. Dorsey G, Njama D, Kamya MR, Cattamanchi A, Kyabayinze D, Staedke SG, Gasasira A, Rosenthal PJ, 2002. Sulfadoxine/ pyrimethamine alone or with amodiaquine or artesunate for treatment of uncomplicated malaria: a longitudinal randomised trial. Lancet 360: 2031-2038.

19. Warrell DA, Molyneux ME, Beales PF, 1990. Severe and complicated malaria. World Health Organization, Division of Control of Tropical Diseases. Trans R Soc Trop Med Hyg 84: 1-65.

20. World Health Organization, 1996. Assessment of Therapeutic Efficacy of Antimalarial Drugs for Uncomplicated Falciparum Malaria in Areas with Intense Transmission. Geneva: World Health Organization.

21. Cattamanchi A, Kyabayinze D, Hubbard A, Rosenthal PJ, Dorsey G, 2003. Distinguishing recrudescence from reinfection in a longitudinal antimalarial drug efficacy study: comparison of results based on genotyping of msp-1, msp-2, and glurp. Am J Trop Med Hyg 68: 133-139.

22. Plowe CV, Djimde A, Bouare M, Doumbo O, Wellems TE, 1995. Pyrimethamine and proguanil resistance-conferring mutations in Plasmodium falciparum dihydrofolate reductase: polymerase chain reaction methods for surveillance in Africa. Am J Trop Med Hyg 52: 565-568.

23. Duraisingh MT, Curtis J, Warhurst DC, 1998. Plasmodium falciparum: detection of polymorphisms in the dihydrofolate reductase and dihydropteroate synthetase genes by PCR and restriction digestion. Exp Parasitol 89: 1-8.

24. Staedke SG, Sendagire H, Lamola S, Kamya MR, Dorsey G, Rosenthal PJ, 2004. Relationship between age, molecular markers, and response to sulfadoxine-pyrimethamine treatment in Kampala, Uganda. Trop Med Int Health 9: 624-629.

25. Plowe CV, 2001. Folate Antagonists and Mechanisms of Resistance. Totowa, NJ: Humana Press.

26. Khalil I, Ronn AM, Alifrangis M, Gabar HA, Satti GM, Bygbjerg IC, 2003. Dihydrofolate reductase and dihydropteroate synthase genotypes associated with in vitro resistance of Plasmodium falciparum to pyrimethamine, trimethoprim, sulfadoxine, and sulfamethoxazole. Am J Trop Med Hyg 68: 586-589.

27. Nzila AM, Mberu EK, Sulo J, Dayo H, Winstanley PA, Sibley $\mathrm{CH}$, Watkins WM, 2000. Towards an understanding of the mechanism of pyrimethamine-sulfadoxine resistance in Plasmodium falciparum: genotyping of dihydrofolate reductase and dihydropteroate synthase of Kenyan parasites. Antimicrob Agents Chemother 44: 991-996.

28. Hastings IM, Watkins WM, White NJ, 2002. The evolution of drug-resistant malaria: the role of drug elimination half-life. Philos Trans $R$ Soc Lond B Biol Sci 357: 505-519.

29. Kamya MR, Bakyaita NN, Talisuna AO, Were WM, Staedke SG, 2002. Increasing antimalarial drug resistance in Uganda and revision of the national drug policy. Trop Med Int Health 7: 1031-1041.

30. Dorsey G, Kamya MR, Ndeezi G, Babirye JN, Phares CR, Olson JE, Katabira ET, Rosenthal PJ, 2000. Predictors of chloroquine treatment failure in children and adults with falciparum malaria in Kampala, Uganda. Am J Trop Med Hyg 62: 686692.

31. Dorsey G, Kamya MR, Singh A, Rosenthal PJ, 2001. Polymorphisms in the Plasmodium falciparum pfcrt and pfmdr-1 genes and clinical response to chloroquine in Kampala, Uganda. $J$ Infect Dis 183: 1417-1420. 